\title{
Arthroscopic Treatment of Lateral Tibial Plateau Fractures: A Simple Technique
}

\author{
Roberto Rossi, M.D., Filippo Castoldi, M.D., Davide Blonna, M.D., \\ Antongiulio Marmotti, M.D., and Marco Assom, M.D.
}

\begin{abstract}
We present a new technique for arthroscopic-assisted fluoroscopic treatment of external tibial plateau fractures (Schatzker type I, II, III), using custom-made instrumentation. The instrument includes 4 basic elements: 2 cutting guides, a hollow trephine cutter with a saw-toothed tip, and a bone plunger. The lateral plateau is restored from the opposite side. An anteromedial rectangular cortical window in the proximal diaphysis of the tibia is created using the cutting guides. To achieve the reduction, the hollow trephine cutter is introduced into the opening created. The bone plunger is inserted into the hollow trephine cutter until resistance is felt. At this point the cancellous bone is compacted by applying force onto the plunger with the hammer so as to obtain a strong compact bone carrot. The plunger then pushes it under the depressed area, filling it out and pushing it upward, creating an indirect reduction. The arthroscopy comes into play estimating the precise anatomic reduction of the articular surface of the tibia. No graft is necessary and cannulated lag screws fix and strengthen the reduction. This new technique is reproducible, easy, less invasive than others, and ensures a stable cartilage and subchondral support without the need for grafts. Key Words: Tibial plateau fracture-Arthroscopy-Fluoroscopy-Technique-Surgical instrumentation.
\end{abstract}

$\mathbf{T}$ ibial plateau fractures represent a complex injury involving the weight-bearing surface of the tibia. Several surgical techniques have been described with the goal of anatomic reduction, rigid internal fixation, and soft-tissue stability. ${ }^{1-4}$ As does ours, most surgical techniques use arthroscopy, which guarantees an accurate diagnosis, provides an assessment of the joint, and follows the reduction, step by step.

From the Third Department of Orthopaedics and Traumatology, University of Turin Medical School, Mauriziano "Umberto I" Hospital, Turin, Italy.

Address correspondence and reprint requests to Roberto Rossi, M.D., Corso Duca degli Abruzzi 15, 10129 Turin, Italy. E-mail: rossir@fastwebnet.it

(C) 2006 by the Arthroscopy Association of North America

Cite this article as: Rossi R, Castoldi F, Blonna D, Marmotti A, Assom M. Arthroscopic treatment of lateral tibial plateau fractures: a simple technique. Arthroscopy 2006;22:678.e1-678.e6 [doi:10.1016/j.arthro.2005.09.028].

0749-8063/06/2206-5164\$32.00/0

doi:10.1016/j.arthro.2005.09.028
Herein, a new, less-invasive surgical technique to facilitate the reduction of the external tibial plateau depression/fractures is proposed. As our experience made apparent the need to resolve several questions in this kind of fracture, such as the filling of the defect, the weakening of the lateral pillar, avoiding further damage to the vascular supply of the lateral plateau, we created a new technique. This procedure has been routinely used in our department since 1998.

\section{SURGICAL INSTRUMENTATION}

The instrument includes 4 basic elements: 2 cutting guides (CGs), a hollow trephine cutter with a sawtoothed tip (HTC), and a bone plunger (BP) (Fig 1). The 2 CGs $(10 \times 20 \mathrm{~mm})$, for right and left tibia, create the window. The CGs have a trapezoid-like shape with 2 small slanting sides, 1 sharp guiding edge, and 1 flat edge. They are used to create a rectangular cortical window with 2 small parallel 

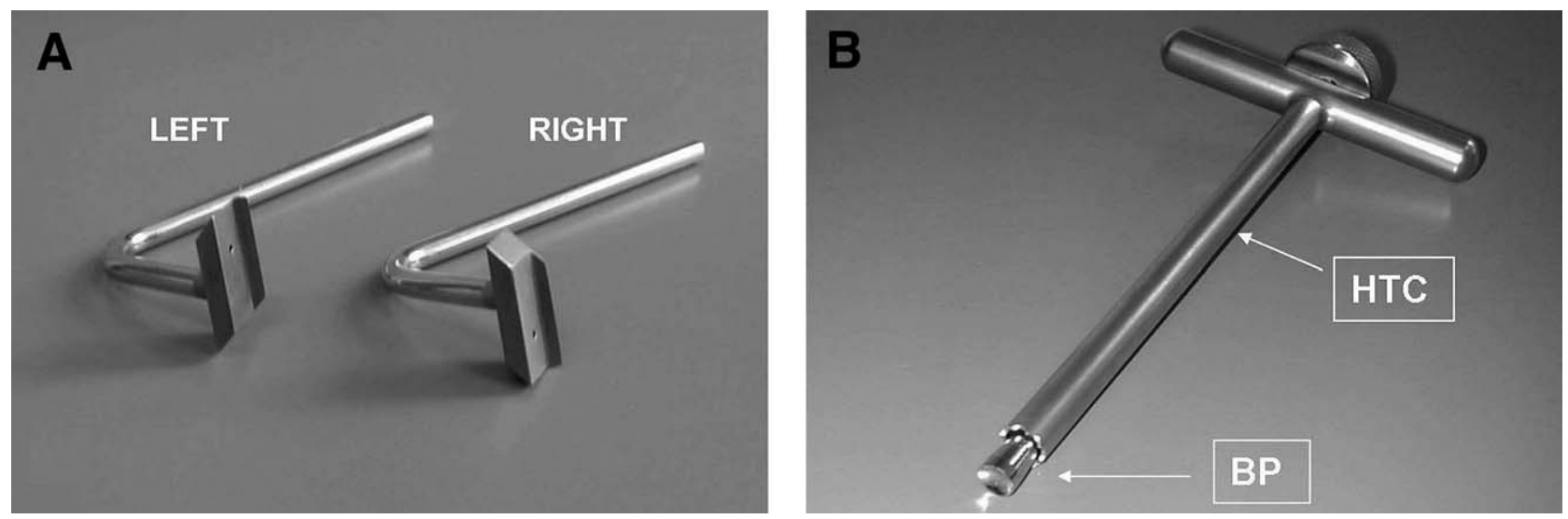

Figure 1. The custom-made instruments include 4 basic elements: (A) 2 (right and left) CGs, (B) an HTC, and a BP.

slanting sides for easy refit. The HTC is cylindrical (10 $\mathrm{mm}$ diameter) with a saw-toothed cutting tip. It collects cancellous bone along its pathway that will be later compressed into a carrot and plunged under the depressed area. The BP is a solid impactor with a concave tip, $9 \mathrm{~mm}$ in diameter. It glides through the HTC and its tip holds, compresses, and drives the cancellous bone carrot under the depressed area.

\section{SURGICAL TECHNIQUE}

First, operative arthroscopy with 2 anterior portals is performed to address any intra-articular pathology. The hematoma and osteochondral fragments are then removed, so the lesion may be assessed and the fracture evaluated. The next step is to create an anteromedial rectangular cortical window with slanting sides, using the CG. First a small skin incision is made and the bone exposed. A small saw is used to make the first cut in the bone, into which the sharp guiding edge of the CG is inserted. At this point, the instrument is held firmly in place and the bone is cut around the guide (Fig 2). The rectangular cortex is then removed and put aside. The HTC is introduced into the opening created under fluoroscopic guidance (2 views), moving in a distal-proximal direction from the opposite side of the depression to about $2 \mathrm{~cm}$ under the depressed area: a cancellous bone carrot is thus obtained. The BP is inserted into the HTC until resistance is felt. At this point, the cancellous bone is compacted by applying force onto the plunger with a small hammer so as to obtain a strong compact bone carrot. The plunger then pushes it under the depressed area, filling it out and pushing it upward, creating an indirect
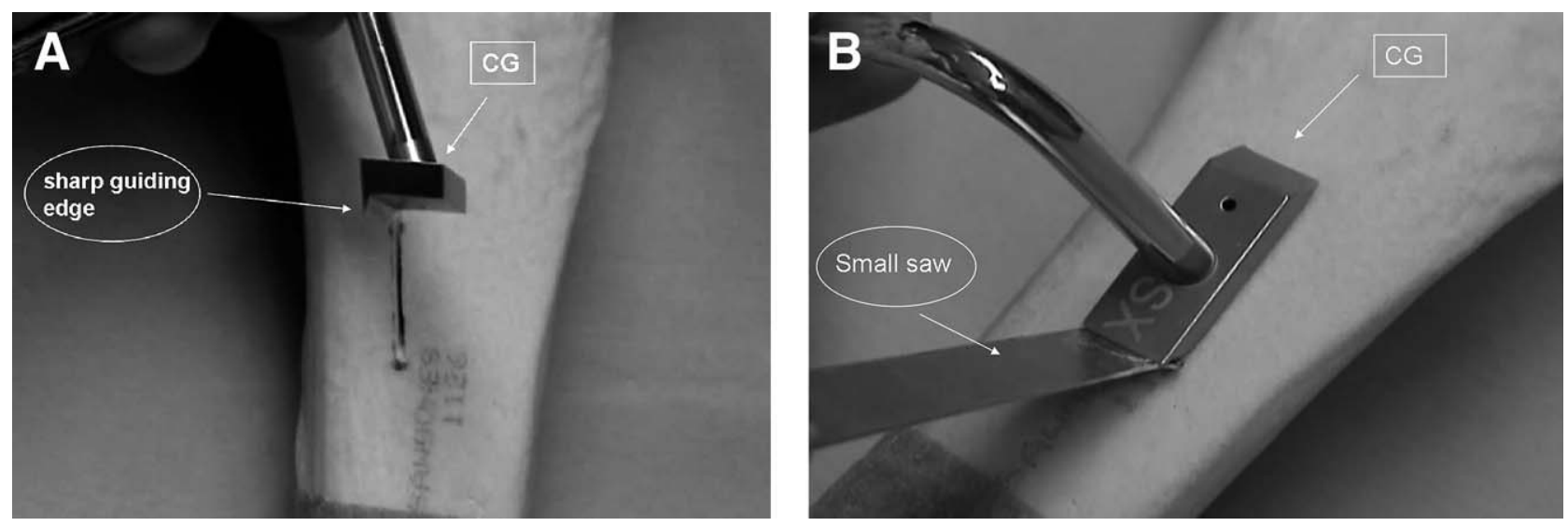

Figure 2. The anteromedial rectangular cortical window with slanting sides, made using the CG, is created. (A) A small saw is used to make the first cut in the bone into which the sharp guiding edge of the CG is inserted. (B) At this point the instrument is held firmly in place and the bone is cut around the guide. 

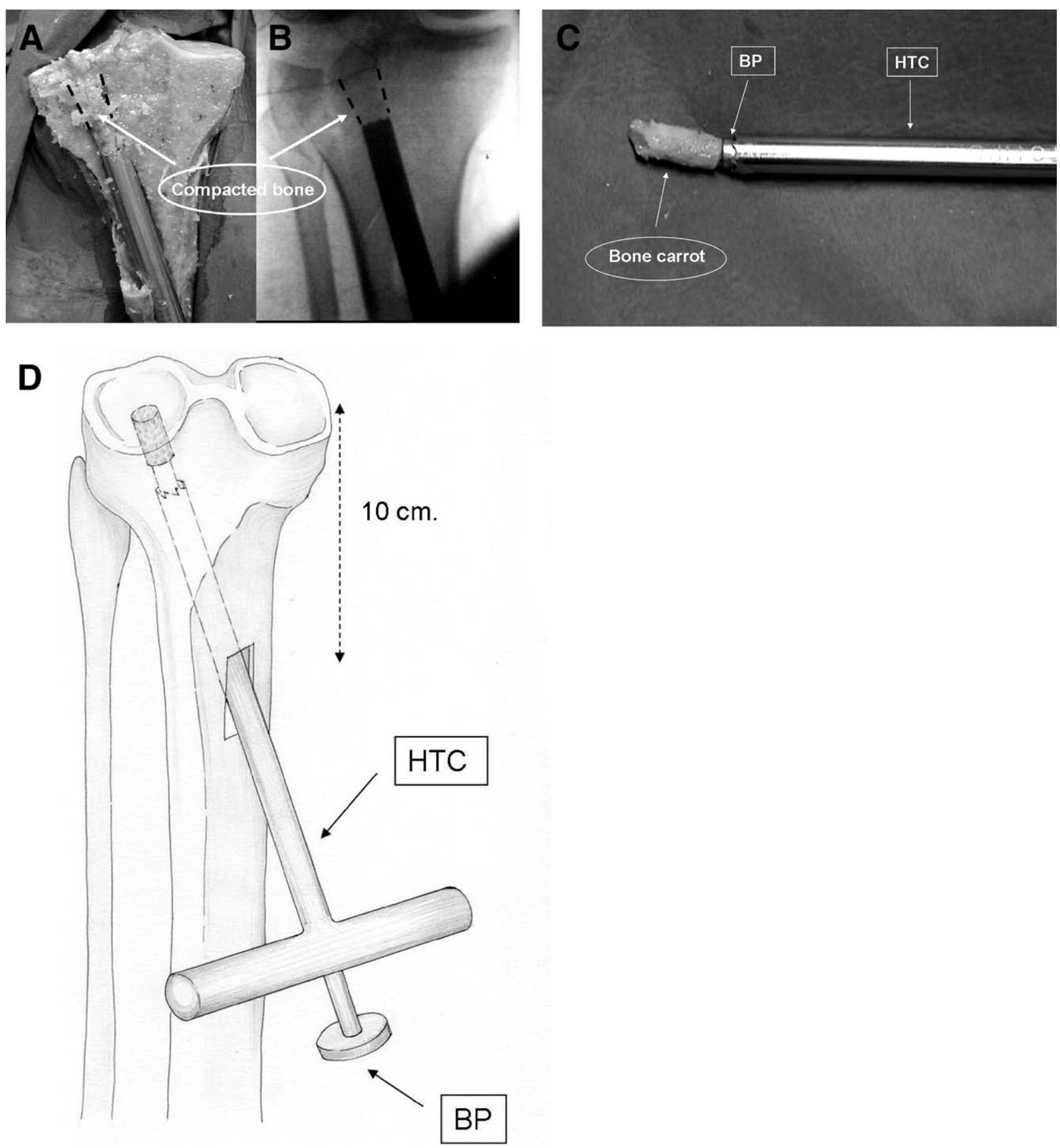

Figure 3. The HTC is introduced into the opening created under fluoroscopic guidance, obtaining a cancellous bone carrot (cadaver specimen). (B) The plunger then pushes it under the depressed area, filling it out and pushing it upward, creating an indirect reduction (fluoroscopic view). (C) The cancellous bone is compacted by applying force onto the plunger with a small hammer so as to obtain a strong compact bone carrot (cadaver specimen). (D) A diagram showing the reduction performed (using the HTC and the BP) indirectly with the bone carrot.

reduction (Fig 3). The whole procedure may be repeated in a different direction through the same window, if needed. Once again, arthroscopy comes into play, estimating the precise anatomic reduction of the articular surface of the tibia.

Last, once the depression has been adequately re- 

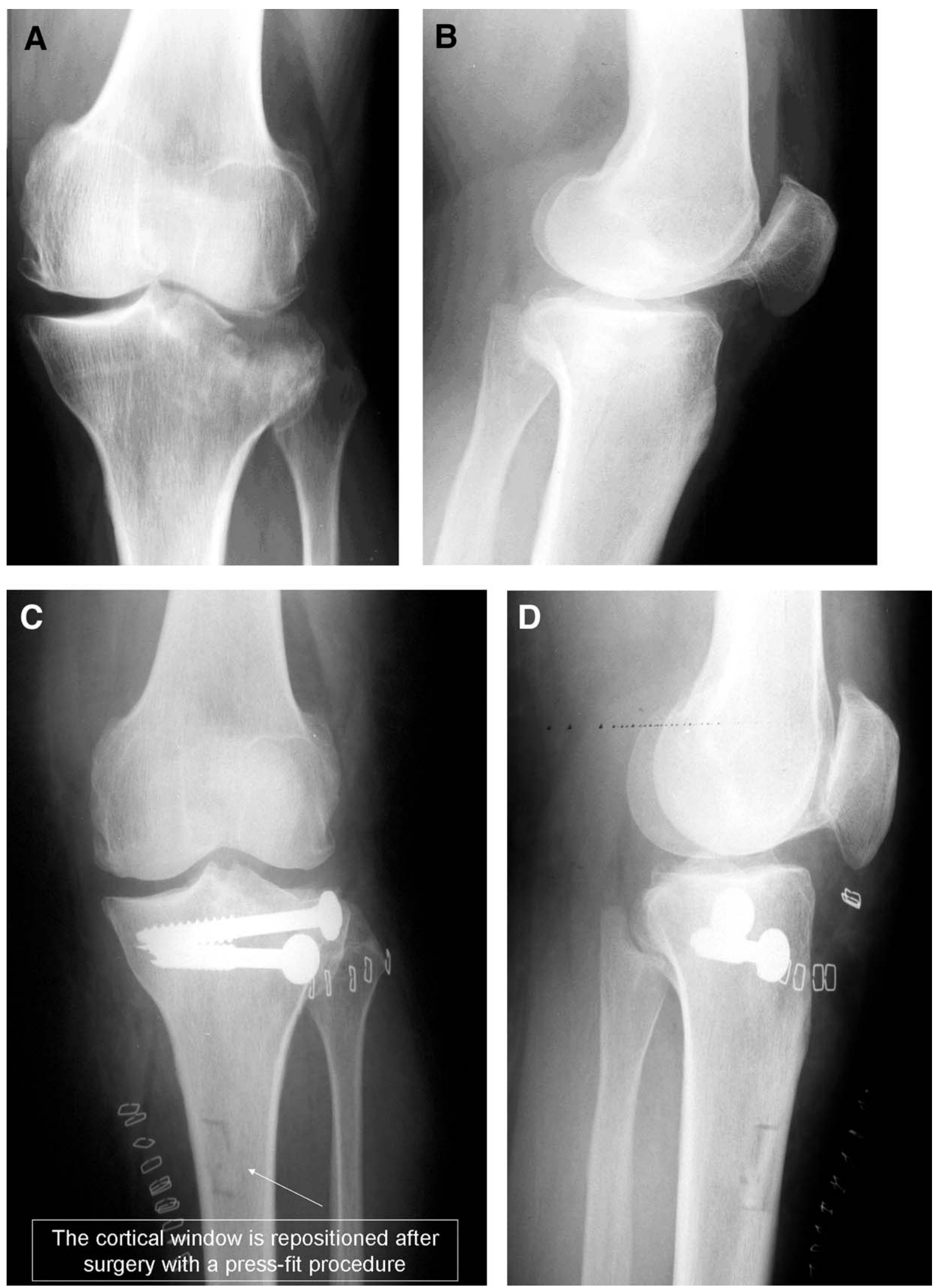

FigURE 4. Preoperative radiographic findings showing a depressed area of the lateral tibial plateau (A: antero-posterior view; B: lateral view). (C,D) Postoperative views showing the anatomical reduction of the depressed area fixed with two cannulated screws (C: anteroposterior view; D: lateral view). 
duced, 2 cannulated lag screws are placed to fix the reduction by passing in a lateral to medial direction, approximately $1 \mathrm{~cm}$ below the articular surface (Fig 4). Postoperative management includes the use of a hinged knee brace, allowing for free motion with a range of $0^{\circ}$ to $90^{\circ}$. After check-up, partial weight bearing is allowed after 8 weeks and a full weight bearing after 3 months.

\section{DISCUSSION}

Currently, arthroscopic reduction and internal fixation is the gold standard for treatment of tibial plateau fracture types I, II, III according to Schatzker classification. ${ }^{2,3,5,6}$ Several clinical studies have shown that the use of the arthroscope gives good results, offering such advantages as the evacuation of hemarthrosis and chondral debris, visualization of the anatomic reduction, a decreased morbidity, reducing hospitalization time, and postoperative care as well as the treatment of associated intra-articular injuries. ${ }^{2-4,6,7}$ Standard practice for the management of the depression and split fractures (types I, II, III of Schatzker classification) of the external tibial plateau use grafts (allograftautograft-substitute-cement) and fixation to maintain the reduction. As a rule, the defect is filled with grafts, to improve articular reduction and decrease the risk of secondary relapse. ${ }^{8}$ Several studies have been published describing reductions with or without grafts. ${ }^{9-12}$ Beaufils et al. ${ }^{8}$ state that, although filling the defect has no statistically significant effect on the results, the outcomes are better when filling is performed, particularly in Schatzker type II fractures. Indeed, any type of graft is able to fill the defect. However, we are of the opinion that autograft should be the first choice.

In the technique herein described, although the graft is taken from the tibia itself, it is far from and contralateral (medial) to the fracture side. A cancellous bone carrot large enough to fill the defect and raise the fracture is obtained by repeating the procedure if necessary, through 1 anteromedial window $10 \mathrm{~cm}$ below the articular surface of the tibia (Fig 3D). In most of the surgical techniques described in the literature, the reduction is performed through the pillar at the same side of the fracture..$^{1,2,13}$

However, this lateral approach may well not only further weaken the fractured meta-epiphysis plateau but also compromise its vascular supply. ${ }^{11}$ That is why we asked ourselves: "why should we compromise the wall just to raise the ceiling?" So we chose to leave the lateral pillar intact by using a medial approach to access the lateral plateau. Research was carried out on cadaver specimens to determine the best access point into the lateral tibial plateau. It was seen that a medial approach offered easy access for the reduction and allowed for the collection of a large quantity of cancellous bone without harming the lateral structures. Indeed, after the tibia had been divided along the frontal plane, it was possible to see the exact amount of impacted cancellous bone relocated under the depression. The site of the cortical window allowed us to obtain a long cancellous bone carrot of about $10 \mathrm{~cm}$ at each procedure. The BP collects the cancellous bone along its path, reducing it into $2 \mathrm{~cm}$ of strong compacted bone carrot, which because of its biomechanical properties is able to support and reduce the depressed area well (Fig 3C).

The HTC device comes in only 1 size $(10-\mathrm{mm}$ diameter) to fit into the cortical window without weakening the proximal anteromedial diaphysis of the tibia, i.e., a larger diameter might well compromise and reduce the maneuverability of the plunger inside the meta-diaphyseal portion of the tibia. With the aim to obtain an indirect reduction, the BP is stopped before the articular surface so to fill and raise the depressed area with the bone carrot. Therefore, there is no need for a precise positioning of the BP under the fracture, thus eliminating the need for any kind of anterior cruciate ligament guide. The fluoroscopic guidance (2 views) makes visibility of the maneuver easy and secure. The cortical window is created directly on the diaphysis of the tibia only to be repositioned after surgery with a press-fit procedure, thanks to its geometry (Fig 4B). Therefore, on the basis of our personal experience and to the best of our knowledge, this is the first time a safe and reproducible reduction of Schatzker type I, II, and III fractures has been obtained with this technique.

Acknowledgment: The authors thank the following people for the critical and specialist support: for editing and reviewing, Barbara Wade, and for the line drawing, Anna Casalone.

\section{REFERENCES}

1. Whittle AP. Fractures of lower extremity. In: Canale T, ed. Operative Orthopaedics. Philadelphia: Mosby, 1998;2094-2111.

2. Buchko GM, Johnson DH. Arthroscopy assisted operative management of tibial plateau fractures. Clin Orthop Rel Res 1996;332:29-36.

3. Jennings JE. Arthroscopic management of tibial plateau fractures. Arthroscopy 1985;1:160-168.

4. Lubowitz JH, Elson WS, Guttmann D. Part I: Arthroscopic management of tibial plateau fractures. Arthroscopy 2004;20: 1063-1070. 
5. Caspari RB, Hutton PM, Whipple TL, Meyers JF. The role of arthroscopy in the management of tibial plateau fractures. Arthroscopy 1985;1:76-82.

6. Gill TJ, Moezzi DM, Oates KM, Sterett WI. Arthroscopic reduction and internal fixation of tibial plateau fractures in skiing. Clin Orthop Rel Res 2001;383:243-249.

7. McClellan RT, Comstock CP. Evaluation and treatment of tibial plateau fractures. Curr Opin Orthop 1999;10:10-21.

8. Beaufils P, Hardy P, Cassard X, et al. Arthroscopic treatment of intra-articular fractures of the knee. Eur Instr Course Lect 2001;5:46-53.

9. Fowble CD, Zimmer JW, Schepsis AS. The role of arthroscopy in the assessment and treatment of tibial plateau fractures. Arthroscopy 1993;9:584-590.
10. Cassard X, Beaufils P, Blin JL, Hardy P. Osteosynthesis under arthroscopic control of separated tibial plateau fracture: 26 case reports. Rev Chir Orthop 1999;85:257-266.

11. Beaufils P, Hardy P, Cassard X. Arthroscopic treatment of intra-articular fractures of the knee. In: Thorngren KG, Soucacos PN, Horan F, Scott J, eds. European Instructional Course Lectures Vol 5. London: The British Editorial Society of Bone and Joint Surgery;2001:46-53.

12. Itokazu M, Matsunaga T. Arthroscopic restoration of depressed tibial plateau using bone and hydroxyapatite grafts. Arthroscopy 1993;9:103-108.

13. Suganumma J, Akutsu S. Arthroscopically assisted treatment of tibial plateau fractures. Arthroscopy 2004;10:10841089. 